\title{
ORAL SESSIONS
}

ORAL SESSION 5D

\section{LIFESTYLE CHANGES AND LIPIDS}

\section{D.01 MORTALITY RISK AND EXERCISE CAPACITY ASSOCIATIONS IN HYPERTENSIVES ACCORDING TO BMI LEVELS}

P. Kokkinos ${ }^{1}$, C. Faselis ${ }^{1}$, J. Myers ${ }^{2}$, T. Manolis ${ }^{3}$, A. Pittaras ${ }^{1}$, F. Kyritsi ${ }^{1}$, M. Doumas ${ }^{1}$, V Papademetriou ${ }^{1}{ }^{1}$ Veterans Affairs Medical Center, Washington, USA, ${ }^{2}$ Palo Alto Health Care Systems, Palo Alto, USA, ${ }^{3}$ Asklepeion General Hospital, Athens, Greece

Objective: To assess the association between fitness, fatness and mortality risk in hypertensive individuals.

Design and Methods: A total of 3,519 men with hypertension ages 6592 years from the VAMC, Washington DC and Palo Alto, California underwent routine exercise tolerance testing. Peak workload was estimated in metabolic equivalents (METs). We established three body weight categories based on BMI levels. Normal weight: BMI <25; Overweight: BMI 25-29.9; and Obese: $\mathrm{BMI}>$ or $=30$. Fitness categories were also established based on peak METs achieved: Low-Fit: $<5$ METs, Moderate-Fit: 5.1-7 METs, Moderate-High: 7.1-9 METs and High-Fit:>9 METs. All-cause mortality is reported within a follow-up period of $8.5 \pm 5.4$.

Results: There were 1403 deaths, with an annual mortality of $4.7 \%$. After controlling for age, risk factors and medications, we observed an inverse and graded association between mortality risk and exercise capacity $(\mathrm{p}<0.001)$. For every 1-MET increase in exercise capacity, the mortality risk for the entire cohort was lowered by $13 \%$ ( $\mathrm{HR}=0.87$; CI: $0.85-0.90$; $\mathrm{p}<0.001)$. For each BMI category the risk was lowered by $9 \%$ for the normal weight individuals; $14 \%$ for the Overweight and $18 \%$ for the Obese. When fitness categories were considered, we observed a graded reduction in mortality risk with increase fitness within each BMI category. The risk reduction was more pronounced for the overweight and obese individuals when compared to those of normal weight.

Conclusion: Our findings support an independent inverse and graded association between fitness levels and mortality risk in individuals with HTN regardless of BMI levels. The impact of fitness on the mortality rate is greater in the overweight and obese individuals compared to those of normal weight.

\begin{tabular}{|l|c|c|c|c|}
\hline & \multicolumn{4}{|c|}{ Fitness Categories } \\
\hline BMI Categories & $<5$ METs & $\mathbf{5 . 1 - 7 . 0 ~ M E T s}$ & $\mathbf{7 . 1 - 9 . 0 ~ M E T s}$ & $>9.0$ METs \\
\hline <25 (Normal Wt) & 1 & $\begin{array}{c}0.80 \\
(0.65-0.98)\end{array}$ & $\begin{array}{c}0.64 \\
(0.47-0.86)\end{array}$ & $\begin{array}{c}0.49 \\
(0.32-0.73)\end{array}$ \\
\hline 25-29.9 (Over Wt) & 1 & 0.68 & 0.50 & 0.37 \\
& & $(0.57-0.82)$ & $(0.37-0.66)$ & $(0.24-0.56)$ \\
\hline 230 (Obese) & 1 & 0.70 & 0.40 & 0.38 \\
& & $(0.57-0.86)$ & $(0.27-0.61)$ & $(0.15-0.92)$ \\
\hline
\end{tabular}
5D.02 RELATIONSHIP BETWEEN OBJECTIVELY MEASURED
PHYSICAL ACTIVTIY AND ARTERIAL STIFFNESS IN HYPERTENSIVE PATIENTS

C. O'Donovan ${ }^{1}$, J. Redmond ${ }^{2}$, T. Raftery ${ }^{2}$, J. Sharma ${ }^{3}$, J. Hussey ${ }^{1}$, J. Gormley ${ }^{1}$, A. Mahmud ${ }^{3}$, F.E. Lithander ${ }^{2} .{ }^{1}$ Department of Physiotherapy,
Trinity College Dublin, Dublin, Ireland, ${ }^{2}$ Department of Clinical Medicine, Trinity College Dublin, Dublin, Ireland, ${ }^{3}$ Department of Pharmacology \& Therapeutics, Trinity College Dublin, Dublin, Ireland

Objective: A lack of physical activity plays an important role in cardiovascular disease, however the mechanism(s) are poorly understood. In addition, the majority of studies which have examined the relationship between physical activity and arterial function have used subjective measures of activity. The aim of this cross-sectional study was to investigate if objectively measured physical activity is associated with arterial stiffness and wave reflection in patients attending an outpatient hypertension clinic.

Design and Method: Physical activity was measured for 7 consecutive days using a triaxial accelerometer (RT3 Stayhealthy). This provides data on estimated energy expenditure in physical activity and time spent in the various intensities of activity. Brachial blood pressure (BP) was measured using an oscillometric technique (Omron) in the right arm. Using the same arm, augmentation index (AIx) and aortic BP were measured using radial applanation tonometry (SphygmoCor). Pulse wave velocity (PWV) was measured using the foot-to-foot method (Artech Medical).

Results: Eighty adults (female, $\mathrm{n}=40$ ) with a mean \pm sd age $49 \pm 25 \mathrm{y}$, body mass index (BMI) $29.7 \pm 5.1 \mathrm{~kg} / \mathrm{m} 2 \quad(\mathrm{n}=70)$ and waist circumference $101 \pm 14 \mathrm{~cm} \quad(\mathrm{n}=42)$ participated. PWV was $10.3 \pm 2.4 \mathrm{~m} / \mathrm{s} \quad(\mathrm{n}=36)$ and AIx was $27.1 \pm 14.4 \%(n=48)$. Physical activity data was available for $\mathrm{n}=53$ patients. Time spent in at least moderate physical activity was $36 \pm 31$ minutes/d and $n=26(49 \%)$ patients carried out at least 30 minutes/d of at least moderate intensity activity. AIx and aortic BP were significantly lower in those who engaged in higher amounts of vigorous activity $(\mathrm{P}<0.01)(\mathrm{n}=48)$. In stepwise regression analysis, after adjusting for age, gender, BMI, alcohol intake and smoking, the only independent predictors of AIx were physical activity, heart rate and systolic BP. There was no relationship between physical activity and PWV $(n=36)$.

Conclusion: Physical activity reduces aortic wave reflections and BP independent of BP and age probably because of increased nitric oxide production in the medium sized arteries. This reiterates the importance of regular physical activity in hypertensive patients to maintain optimum wave reflections.

\section{D.03 THE IMPACT OF DYNAMIC RESISTANCE TRAINING ON BLOOD PRESSURE AND OTHER CARDIOVASCULAR RISK FACTORS: A META-ANALYSIS OF RANDOMIZED CONTROLLED TRIALS}

V. Cornelissen ${ }^{1}$, R.H. Fagard ${ }^{2}$, L. Vanhees ${ }^{1} .{ }^{1}$ K.U.Leuven, Department of Rehabilitation Sciences, Leuven, Belgium, ${ }^{2}$ K.U.Leuven, Department of Cardiovascular Diseases, Leuven, Belgium

Objective: To examine the effect of dynamic resistance exercise training on blood pressure (BP) and other cardiovascular risk factors in healthy sedentary adults.

Design and Methods: Meta-analysis of studies retrieved from four electronic databases as well as cross-referencing from identified articles. Inclusion criteria were as follows: randomized controlled trials; dynamic resistance training of at least 4 weeks was the sole intervention; participants were sedentary normotensive and/or hypertensive adults ( $i Y 18$ years) with no other concomitant disease; resting systolic and/or diastolic BP were available; and finally the article was published in a peer-reviewed journal up to July 2009. Random effects models weighting for the number of trained participants were used for analysis with data reported as means and $95 \%$ confidence limits (CL).

Results: 25 randomized controlled trials were included, involving 29 study groups (sg) and 845 men and women (368 controls, 477 exercisers). According to baseline BP, 11 study groups were classified as normotensives, 14 and 4 as prehypertensives and hypertensives, respectively. Resistance training induced a significant net BP reduction of -2.2 (95\% CL, $-4.3 ;-0.23)$ / $-3.0 ;(-4.2 ;-1.7) \mathrm{mmHg}$; the $\mathrm{BP}$ responses were not significantly different between the three subgroups $(\mathrm{P}>0.25)$. In addition, resistance training significantly increased peak $\mathrm{VO} 2(+3.2(+1.5 ;+4.8) \quad$ mL.min-1.kg-1 $(\mathrm{n}=8 \mathrm{sg})$, reduced body fat by $0.46(-0.86 ; 0.053) \%(\mathrm{n}=11 \mathrm{sg})$ and reduced 